28 | 2002

Histoire de l'enseignement du français langue

étrangère ou seconde dans le bassin méditerranéen.

Volume 2

\title{
Charles-Louis-Jules David, professeur de français à Chio
}

Jean Antoine Caravolas

\section{QpenEdition \\ Journals}

Édition électronique

URL : https://journals.openedition.org/dhfles/2658

DOI : $10.4000 /$ dhfles.2658

ISSN : 2221-4038

Éditeur

Société Internationale pour l'Histoire du Français Langue Étrangère ou Seconde

Édition imprimée

Date de publication : 1 juin 2002

Pagination : 87-101

ISSN : 0992-7654

Référence électronique

Jean Antoine Caravolas, "Charles-Louis-Jules David, professeur de français à Chio », Documents pour l'histoire du français langue étrangère ou seconde [En ligne], 28 | 2002, mis en ligne le 31 janvier 2014, consulté le 27 mai 2021. URL : http://journals.openedition.org/dhfles/2658 ; DOI : https://doi.org/ $10.4000 /$ dhfles.2658

Ce document a été généré automatiquement le 27 mai 2021.

(c) SIHFLES 


\title{
Charles-Louis-Jules David, professeur de français à Chio
}

\author{
Jean Antoine Caravolas
}

\section{Le fils du peintre}

1 Jules David ${ }^{1}$ est né à Paris en 1783. Il était le fils aîné du peintre Louis David (1748-1825) et de Charlotte Pécoul (1765 7-1826). Louis David était ami de Robespierre et comme député à la Convention il vota la mort du roi. Charlotte était fille de l'entrepreneur des Bâtiments du Roi, et royaliste. Pendant la Révolution le couple traverse une crise profonde et en 1794 divorce pour se remarier en 1795, à la sortie de l'artiste de prison, où les thermidoriens l'avaient enfermé par deux fois. Sous Napoléon, Louis jouit des faveurs de l'empereur, dont il devient le peintre. David atteint alors le sommet de sa gloire. Son œuvre est célébrée et imitée dans toute l'Europe. À la Restauration toutefois, il est de nouveau poursuivi et doit s'exiler à Bruxelles, où il meurt le 29 décembre 1825. Charlotte meurt six mois plus tard à Paris.

2 Jules reçut une solide éducation classique au Prytanée (le collège Louis le Grand) et ensuite à l'université de Göttingen en Allemagne (Troyat 1990 : 147), où les études classiques, en particulier grecques, connaissent alors un renouveau spectaculaire grâce notamment aux néo-humanistes. K. M. Gesner et Chr. G. Heyne (J. Caravolas, $2000: 114$, 145-148). À son retour d'Allemagne il est nommé auditeur au Conseil d'État, vice-consul à Civita-Vecchia (1805-1808), vice-consul à Otrante (1808), sous-préfet à Stade (Bouches de l'Elbe) de 1810 à 1814 et, en 1815, préfet d'un département en France. Après la bataille de Waterloo, il va vivre avec ses parents à Bruxelles, mais il n'y reste pas longtemps.

3 Alors que sa mère s'active pour lui obtenir un poste de professeur de grec en Belgique, Jules revient à Paris et au mois d'août 1816 se laisse recruter par le père des Lumières grecques Adamantios Coray(s) ou Koraiis (1743-1833), qui cherche des professeurs de qualité pour le célèbre gymnase de Chio. Quelques jours plus tard, Coray reçoit de Bruxelles une lettre de Mme David le priant de lui faire parvenir un certificat attestant 
la compétence de son fils en grec, afin qu'il puisse l'enseigner en Belgique. Mais Jules était déjà en route pour la Grèce.

4 En quittant Paris, le " fils du peintre " ne descend pas à Marseille prendre le bateau, comme il lui avait été recommandé. Craignant les dangers de la mer, il préfère se rendre dans la patrie d'Homère ${ }^{2}$, sans trop se hâter, via Vienne et Constantinople, ainsi ne débarque-t-il au port de Chio que le 10 décembre 1816. Jules est enchanté par l'accueil que lui réservent le principal de l'école et les notables de la ville ${ }^{3}$, se familiarise vite avec le milieu grec, commence, sans perdre de temps, ses cours de français, prend des notes sur le grec moderne pour les livres qu'il songe à écrire et épouse une jeune grecque (Grand Dictionnaire universel [...] 1990 : 162 ; Bäsch $1995: 61)^{4}$ Soudain, quelque

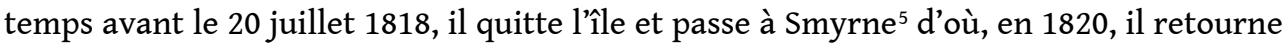
en Europe ${ }^{6}$. À Paris, en attendant un poste à l'université, il reprend ses recherches sur les rapports du grec moderne avec le grec ancien et sur la manière de les enseigner et de les apprendre. Mais les portes de la Sorbonne restent fermées au fils du régicide. Quand finalement, en 1831, elles s'entrouvrent, on ne lui accorde que la charge de " suppléant du professeur Boissonnade » (1774-1857), helléniste, membre de l'Institut et ami de Coray. Au sujet de son enseignement à l'université il écrira plus tard :

M. David rappelle, comme un de ses principaux titres, qu'il a été suppléant de M. Boissonnade ${ }^{7}$ à la chaire de littérature grecque de la Faculté des lettres, et qu'il a exercé ces fonctions pendant dix ans ; il croit s'en être acquitté de manière à donner une idée favorable de son instruction dans la langue grecque (David 1853 : 3).

5 Jules David mourut en 1854. Selon le Grand Dictionnaire universel du XIXe siècle, « jusqu'à la fin de sa vie il resta fidèle au culte de la Révolution, dont il avait, si l'on peut dire, sucé les principes avec le lait, et il n'avait jamais eu la fantaisie de porter le titre de baron que son père avait reçu de Napoléon ${ }^{8} »(1990: 162)$.

\section{L'helléniste}

6 Jules naît à la veille de la Révolution française, mais aussi au moment où en Europe se développent le néo-classicisme et le néo-humanisme, mouvements qui prônent le retour aux valeurs et aux modèles de l'Antiquité, surtout grecque, dans les arts et en éducation. Ces deux tendances culminent en France sous le Directoire (1795), le Consulat (1799) et l'Empire (1804-1815) et prennent la forme d'une " grécomanie » qui se manifeste en particulier à Paris, et cela dans tous les domaines de la vie privée et publique : mode, repas, meubles, architecture, sculpture, peinture, poésie, théâtre, éditions et rééditions de récits de voyages, de livres savants ou d'ouvrages littéraires qui ont un rapport avec la Grèce, notamment Les Aventures de Télémaque (1699) de Fénelon et Le Voyage du jeune Anacharsis en Grèce (1788) de l'abbé Barthélémy.

7 A la même époque, la petite colonie grecque de Paris, avec Coray en tête, profite de l'engouement des Français pour la Grèce antique et réussit, par ses contacts personnels, ses activités professionnelles et ses interventions dans la presse, à étendre l'intérêt d'un nombre considérable de représentants de la noblesse (duc d'Orléans, duc de Choiseul), de l'armée (le général Sebastiani), de la finance (Eynard), du monde des arts et des lettres (Chateaubriand, Victor Hugo, Delacroix) à la Grèce moderne. Ainsi voit le jour un vaste mouvement philhellène qui, outre son appui à la lutte des Grecs pour leur 
émancipation culturelle et politique (Société française philanthropique en faveur des Grecs), encourage également l'étude de la langue grecque moderne ${ }^{9}$.

Jules avait étudié le grec ancien au Prytanée et ensuite à l'université de Göttingen. Il avait acquis les rudiments du grec moderne, probablement en 1800, dans les cours de Villoison à l'École des nouvelles langues orientales à la Bibliothèque Nationale à Paris. Il se perfectionna en cette langue par la pratique, durant son séjour à Chio et à Smyrne et en épousant une Grecque. C'est également à Chio qu'il se mit à travailler sur le Parallèle des langues grecques ancienne et moderne (1820), la Méthode pour étudier la langue grecque moderne (1821b) et sur le Dictionnaire français et grec ancien.

Le Parallèle des langues grecques ancienne et moderne est rédigé par l'auteur en grec moderne et s'adresse à des philologues et plus généralement à des lecteurs avancés en grec ancien. Il fut bien accueilli par la presse (Classical Journal 1825 : 152-159), Logios Ermis (Le Mercure savant) 1821 : 155-165), traduit en anglais par John Mitchell et publié à Londres en 1824. David décrit son livre ainsi :

Cet ouvrage, destiné aux personnes qui connaissent déjà le grec ancien et qui désirent s'y perfectionner, est écrit en grec moderne, mais dans le style classique en sorte qu'il est intelligible aux personnes versées dans le grec ancien. Il est propre à leur servir de guide pour acquérir une idée assez exacte du grec moderne, afin de tirer de cette étude tout ce qui peut compléter les connaissances helléniques. Il répond d'une manière positive à ces questions si souvent adressées aux Grecs sur la ressemblance entre leur langue et le grec ancien. On peut s'y convaincre que cet idiome nouveau est beaucoup plus rapproché de l'ancien que l'italien l'est du latin, et que cette ressemblance s'accroît tous les jours par le développement naturel et raisonné qu'amène une éducation éclairée et bien dirigée. (David $1853: 1$ )

10 La Méthode pour étudier la langue grecque moderne (1821b) est par contre conçue pour le grand public et pour cela rédigée en français. Le but de l'ouvrage est de « fournir les moyens de l'étudier [la langue moderne] aux personnes qui n'ont aucune connaissance de l'hellénique ou grec ancien » (Préface : iv). L'auteur se limite pour cela « à la langue usitée», mais renvoie ses lecteurs plus exigeants à son premier livre, car il est convaincu " qu'en général, le seul moyen de se perfectionner dans l'étude du grec moderne, c'est de connaître l'ancien, tant pour la grammaire que pour le dictionnaire » (Ibid. : vj).

11 Le livre compte 150 pages, dont 103 sont consacrées à la grammaire, que David divise en 63 petits chapitres. Les trois premiers traitent de la prononciation, de l'accent et des signes en usage dans l'écriture, les chapitres iv-xxvde la morphologie et les derniers (xxvi-lxiii) de la syntaxe du grec moderne. Les dix-huit pages suivantes (104-122) sont réservées à six dialogues sur des sujets traditionnels, en grec moderne avec en parallèle la traduction française. Ils sont violemment anti-turcs et anti-anglais. En revanche, ils présentent le consul de France à Smyrne dans les termes les plus flatteurs [pp. 108-109. Voir encadré à la page suivante]. 


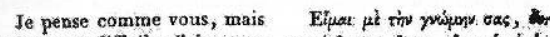

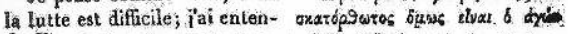

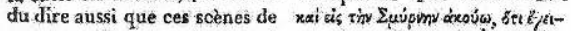

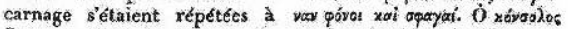

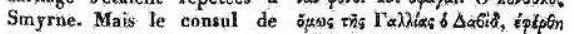

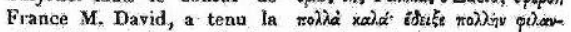

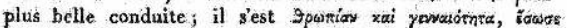

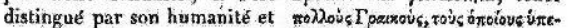

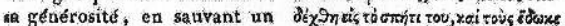

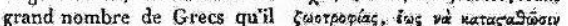

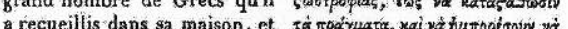
recueills dans sa cur a far de

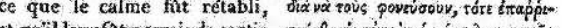

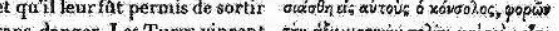

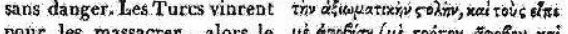

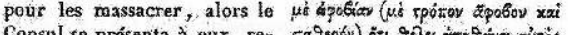

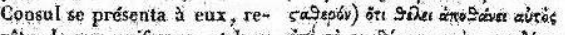

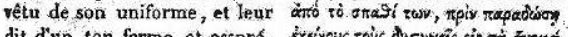

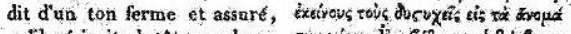

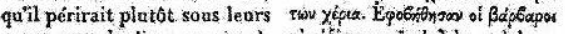

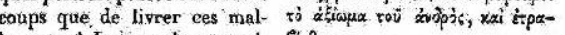
heureux a leurs mains sangui- bixonrav. paires. La fermeté de ce ma-

gistrat en imposa aux barbares

et ils se retirèrent.

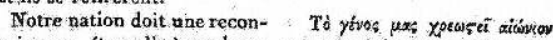

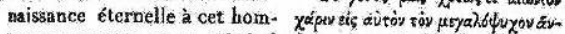

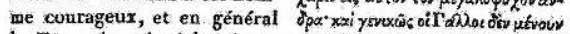

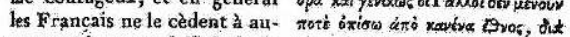

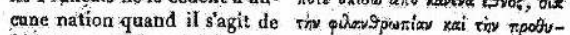
Gire prear

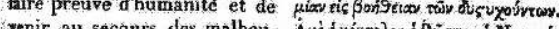

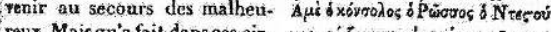

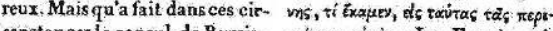

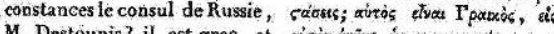

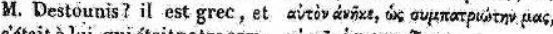

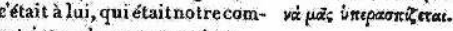
patriote, de nous protéger.
\end{abstract}

Le manuel finit avec l' «Onomasticon ou Liste de mots les plus usuels, par ordre de manières » (pp. 123-150), en français et en grec. En voici quelques exemples :

\section{Le Dessert. \\ Tá ơr}

La pomme, ró $\mu$ ñरov, ov. le pom-

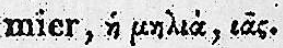

La poire, tò ámidt, coú. le poirier,

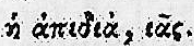

La pêche, ró poớáxevov, ou. le pêcher,

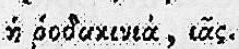

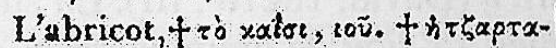

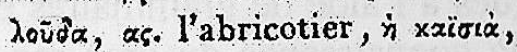

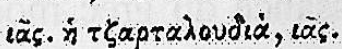

La cerise, tò xspácr, toü. le cerisier, मे xepactín,

La figue, rò oüxov, av. le figuier, ÿ ouvista, $t \bar{\alpha} s$.

La prune, tó dapáaronvos, ov. le pra-

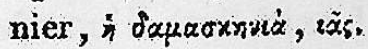

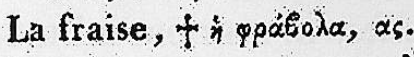

\section{Degreis de parente.}

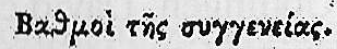

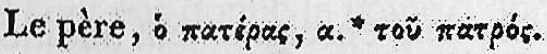

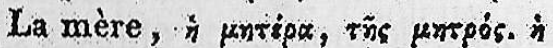
$\mu \dot{\alpha} v y \alpha, \alpha s:$

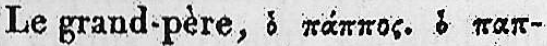
moùins, n.

La grand-mere, in трориitup, opos.

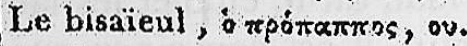

Lie fils, $\delta$ vilos, ov.

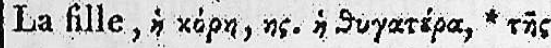
Iuyarpós.

Le frère, ó àdikpos, oũ. - utérin,

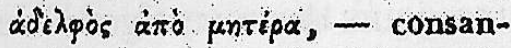
guin, áró rarépa. le frère et la sour, les deux frères, rá ádrìqua.

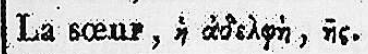


David était très fier de cet ouvrage et rappelait à ses lecteurs « qu'il était approuvés des Grecs, très répandu en Grèce, et fort utile aux étrangers; ceux-ci n'ont pas d'autre guide dans l'étude de la langue parlée » (David 1853 :2). En effet, la Méthode [...], revue et corrigée par l'auteur, fut rééditée à Paris en 1828 ; traduite en anglais par George Winnock et publiée à Londres (1825) et à Oxford (1826), traduite en allemand par Wilhelm von Lüdemann et publiée à Leipzig (1828). L'édition anglaise parut toutefois sans les dialogues de David. Le traducteur, les ayant jugés trop tendancieux, les remplaça par des phrases extraites d'ouvrages d'auteurs grecs et français.

Quant à son Dictionnaire français et grec ancien ${ }^{10}$, " fruit de 25 ans de travaux», David déclare en 1853, qu'il « est terminé depuis longtemps ; mais il est encore inédit, parce que M. David n'a pas les fonds nécessaires pour entreprendre une publication si considérable. "N'étant pas moi-même helléniste, je ne puis me prononcer sur l'importance de ses traités philologiques, les spécialistes toutefois s'accordent sur leur solidité et utilité ${ }^{11}$ (Triandafyllidis $1938: 617$, Schinas : 1829, Legrand $1878: \mathrm{X}$ ).

Dans tous ces ouvrages on sent l'influence des théories linguistiques des néohumanistes allemands, en premier lieu de C. G. Heyne qui fut son maître ${ }^{12}$ à l'université de Göttingen. David croit que l'étude des langues doit servir aussi bien la culture du goût que les besoins pratiques des apprenants. Il rejette pour cela la méthode d'enseignement traditionnelle qui ne fait, selon lui, que « charger la mémoire de mots et de phrases sans acquérir une idée de plus. " Il croit que l'apprentissage deviendra bien plus simple lorsque « les hommes ne parleront qu'une langue», quand il suffira « pour s'entendre d'un pôle à l'autre, d'une seule grammaire et d'un vocabulaire " (David, 1821b : HI). En attendant cependant, il faut, dit-il, étudier les langues à l'aide de grammaires et de dictionnaires. Enfin, il est convaincu que la connaissance du grec moderne est nécessaire à l'apprentissage du grec ancien et inversement, car non seulement on y trouve les mêmes mots avec le même sens, mais aussi la prononciation des deux langues ${ }^{13}$ est en grande partie la même et la syntaxe du grec moderne maintient toujours des « vestiges » de la syntaxe de l'ancien.

Pourtant l'étude du grec moderne est, ajoute l'auteur, négligée par les étrangers. Cela arrive parce qu'ils ne connaissent de cet idiome que ce qu'en racontent les touristes occasionnels, qui eux-mêmes tiennent leurs informations des " Frangoi » (surnom donné par les Grecs aux Européens qui vivent en Grèce), " les ennemis les plus implacables » des Grecs (David, $1820:$ i) et de missionnaires catholiques ignorants, surtout italiens. Certes, on rencontre en Occident des savants sans préjugés, mais soit ils n'ont jamais mis le pied en Grèce, comme Du Cange, soit ils ne se sont pas donné la peine d'apprendre le grec moderne, ou encore ils appartiennent à la catégorie d'auteurs qui écrivent pour gagner de l'argent plutôt que pour faire avancer la science $^{14}$. Les travaux des érudits grecs sont tout aussi insatisfaisants, car ils s'intéressent plus à la réforme de l'orthographe ou à la création d'une nouvelle langue grecque, qu'à l'élaboration de la grammaire de la langue néo-hellénique existante.

\section{David professeur de français à Chio}

17 Jules David partit de Paris le 24 août 1816. Auparavant il passa plusieurs jours en compagnie de Coray, qui voulait l'observer de près, afin d'être absolument sûr qu'il était bien l'homme qu'il fallait pour le poste de $\mathrm{Chio}^{15}$ et aussi pour lui montrer la méthode lancastrienne ${ }^{16}$ que Jules devait suivre dans son enseignement au gymnase. Tout 
indique que le « sage Grec » resta entièrement satisfait de sa rencontre avec « le fils du peintre " et qu'il fut fort impressionné par son impatience de contribuer à la renaissance culturelle de la Grèce. Coray attendait donc beaucoup de David, mais parallèlement il prévenait ses compatriotes que "l'heureux accouchement " [sic] dépendra principalement de l'habileté avec laquelle les Chiotes, en premier lieu les professeurs, le traiteront lorsque l'étranger sera sur place (Coray, 1982 : 494).

Quand le 10 décembre 1816, vers minuit, Jules arriva enfin à sa destination, Chio était encore 1'« île des vins...] qu'ombrageaient les charmilles/Chio, qui dans les flots, reflétait ses grands bois,/ ses coteaux, ses palais, et le soir quelquefois/ un chœur dansant de jeunes filles » (V. Hugo : 1828/1961 : 115). Profondément touché par l'accueil des Chiotes et en guise de remerciements pour toutes leurs bontés, Jules demanda qu'on lui permette d'entamer, sans perdre de temps, l'enseignement de la " philologie française » en se servant de la méthode de Lancaster, comme le lui avait recommandé Coray.

Le principal du collège le lui déconseilla cependant. Il jugeait que le temps n'était pas encore propice, l'école n'ayant pas les dimensions nécessaires pour l'introduction de cette méthode [il faut un espace pouvant contenir jusqu'à mille élèves] et afin de ne pas éveiller les soupçons des Turcs [la méthode demande une organisation et une discipline presque militaires $\left.{ }^{17}\right]$. Par contre, il l'encouragea à suivre provisoirement une approche plus traditionnelle et à concentrer toute son attention sur l'enseignement des fondements de la langue française ${ }^{18}$.

20 David dut donc tout repenser. Deux semaines après son arrivée sur l'île, il avait déjà établi son nouveau plan d'action et pouvait affronter ses élèves. Apprenant que dans tout le gymnase il n'y avait pas un garçon qui parlât français, il résolut d'enseigner d'abord les rudiments de la langue et de laisser la " philologie " pour plus tard. Il choisit à cet effet, pour commencer, trente garçons familiarisés quelque peu avec la terminologie grammaticale de l'ancien grec afin, qu'en cas de besoin, il puisse poursuivre ses explications en cette langue qu'il maîtrisait alors bien mieux que le grec moderne. Puis, pour motiver ses élèves, il les réunit dans une salle à laquelle étaient invités aussi les adultes qui voulaient bien l'entendre, et se mit à leur parler en grec des avantages de la langue française et de son utilité pour les Grecs.

21 Ensuite, il assura son auditoire que les meilleurs prédicateurs (Bossuet, Massillon), les meilleurs poètes (Corneille, Racine, Molière, La Fontaine, Boileau) et les meilleurs écrivains (Montesquieu, Rousseau) étaient français ; que la langue française était admirée par tous les gens éduqués et par les rois d'Europe pour sa clarté, sa précision et sa syntaxe naturelle, si " semblable » à la syntaxe grecque ; qu'elle tenait, à cause de cela, le rôle de langue universelle ; que les traités internationaux étaient rédigés en français et qu'enfin, la connaissance du français aiderait les Grecs à épurer leur langue moderne (David $1820: 141$-144). 


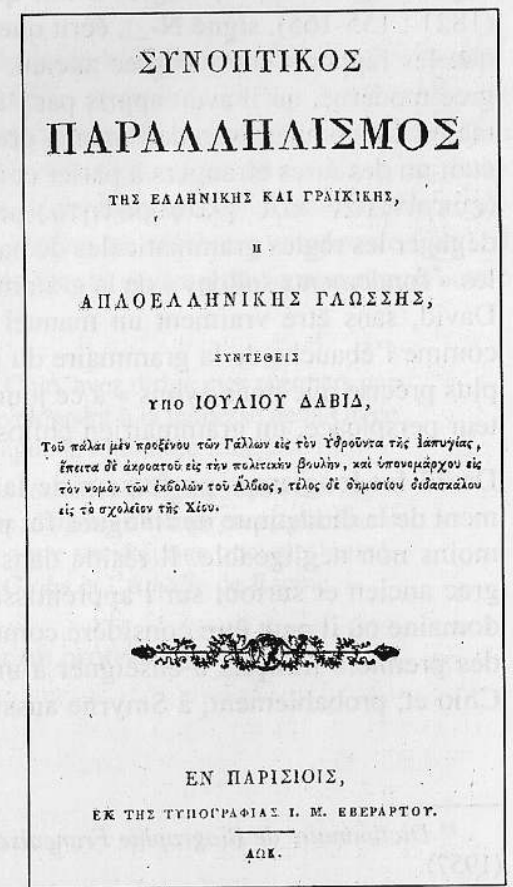

Je ne connais pas le manuel dont David se servit pour enseigner les rudiments de français, ni les détails de la méthode qu'il utilisa. Ce qui est sûr qu'il sut exploiter la soif d'apprendre des jeunes Chiotes et qu'au bout d'un mois il les rendit, à en l'en croire, capables de traduire, de faire des thèmes et d'expliquer un chapitre et demi des Aventures de Télémaque! Encouragé par ces succès, il ouvrit alors une deuxième classe de débutants, tandis qu'avec les élèves les plus avancés il abordait las finesses de la langue (la « philologie ») et les auteurs.

Ravi des progrès rapides de ses étudiants, Jules écrivit le 23 février une longue lettre enthousiaste à Coray, en grec moderne, pour l'informer de ses impressions de Chio, lui décrire la méthode qu'il utilisait dans son enseignement et aussi pour le remercier de lui avoir fourni l'occasion de servir la Grèce " sacrée » (Coray, 1984 : 16-18). Pourtant, un an plus tard, il quitta Chio, comme nous l'avons déjà dit, et se rendit à Smyrne d'où, en 1820, il retourna à Paris. Là, il passera ses jours à écrire ses livres, à enseigner le grec ancien et le grec moderne et à œuvrer, jusqu'à son dernier souffle, en faveur de sa chère Hellade, entretemps devenue indépendante.

\section{Conclusion}

24 Jules David, était un helléniste respecté. Son Parallèle des langues grecques ancienne et moderne fut en son temps bien accueilli des spécialistes et encore aujourd'hui occupe une place importante dans l'histoire de la philologie. La revue Logios Ermis qui lui consacra un long compte rendu (1821:155-165), signé N-., écrit que dans cet ouvrage l'auteur démontre que les rapports entre le grec ancien, qu'il avait longuement étudié, et le grec moderne, qu'il avait appris par l'usage, étaient abondants et incontestables. La comparaison de l'ancien grec avec le grec moderne, dont David était un des rares 
étrangers à parler et à écrire avec " justesse et élégance " (eukrineain kai glafuroteta) permit au " philhellène français » de dégager les règles grammaticales de base du grec moderne et de poser ainsi les« fondements solides » de la grammaire néo-hellénique. Bref, le livre de David, sans être vraiment un manuel de grammaire, peut être considéré commel'ébauche de la grammaire du grec moderne « la plus parfaite et la plusprécise que nous ayons " à ce jour et son auteur comme "un observateurperspicace, un grammairien philosophe et un excellent philologue. »

David futlongtemps professeur de langues, mais son apport à $\mathrm{F}$ avancement de la didactique des langues fut plutôt modeste et peu original, néanmoins non négligeable. Il réside dans ses travaux sur l'enseignement du grec ancien etsurtout sur l'apprentissage du grec moderne aux Français, domaine où il peutêtre considéré comme un pionnier. D'une part il fut un des premiersfrançais à enseigner à un nombre considérable de Grecs (à Chio et,probablement, à Smyrne aussi) ${ }^{19}$ le français de manière systématique. D'autre part les dialogues et d'« onomasticon » de sa Méthode pour étudier la langue grecque moderne pouvaient, au besoin, être utilisés pour apprendre à tenir en français une conversation simple, sur des sujets de la vie quotidienne.

Bien que court, le séjour de David en Grèce laissa une empreinte profonde et durable. Les Grecs n'oublièrent jamais le fils du peintre de Leonidas aux Thermopyles; l'auteur de l'Appel passionné aux Nations en faveur des Grecs (1821a) soulevés en 1821 pour leur indépendance ; le Français qui, croyant que « la liberté d'un peuple est l'affaire de tous »(Ibid.), accourut aux temps les plus difficiles de leur histoire moderne transmettre ses connaissances à la jeunesse de Chio ; l'érudit qui contribua avec ses livres à la renaissance culturelle de leur pays. Pour tout cela Logios Ermis, la revue culturelle grecque la plus prestigieuse de l'époque, écrivait déjà le 3 février 1821 que David méritait bien d'être « naturalisé citoyen Grec. »

Le Grec qui garda le plus vif souvenir de "Monsieur David " fut probablement Panayotis Soutsos (1806-1868), rejeton de la famille princière du même nom et principal représentant de la poésie romantique grecque. En 1828, se trouvant à Paris, où il publie les Odes d'un Jeune Grec, en français, il se rappelle que quand, à l'âge de douze ans ses tuteurs l'envoyèrent avec son frère aîné Alexandre étudier au célèbre gymnase de Chio, il eut pour professeur de français Jules David et il lui dédia son recueil avec ces mots

[...] vous qui au gymnase de Chio, avez dirigé mes premiers pas sur le Parnasse français, en développant à la jeunesse de la Grèce le goût de l'élégance qui distingue la littérature française [...].

J'ai composé ces poésies dans les cafés d'Égine, sur les rivages plaintifs de la déserte Salamine, dans les champs ensanglantés de Marathon, n'ayant pour toute autre société, que quelques numéros de l'excellent Journal du Globe et l'Atlialie de Racine.

Quelle plus grande satisfaction pour un professeur? 


\section{BIBLIOGRAPHIE}

ARMONIA(1900). T. 1. Athènes.

BARTHELEMY, abbé (1788) : Voyage du jeune Anacharsis en Grèce dan le milieu du IV siècle vulgaire.

Paris, de Bure l'aîné.

BASCH, Sophie (1995) : Le mirage grec. La Grèce moderne devant l'opinion française (1846-1946).

Confluences. Paris, Hatier.

CARAVOLAS, Jean Antoine (2000) : Histoire de la didactique des langues au siècle des Lumières. Précis et anthologie thématique. Les Presses de l'université de Montréal. Tübingen, Gunter Narr Verlag.

CLASSICAL JOURNAL(1825). Londres

CORAY(S), Adamantios (1982) : Allilografia [Correspondance] Vol 3 ; (1984) : Allilografia. vol. 4. Athina, Vivliopolion Ermis.

DAVID, Jules (1820) : Synoptikos Parallilismos tis Ellinikis kai Graikikis, i aptoelli-nikis glossis [Parallèle des langues grecques ancienne et moderne] En Parisiis, Ek tis typografias I.M. Everartou.

DAVID, Jules (1821a) : Appel aia nations en faveur des Grecs. Paris, Chez les Marchands de nouveautés.

DAVID, Jules (1821b) : Méthode pour étudier la langue grecque moderne. Paris, Lequien, libraire. [1828]. Nouvelle édition, revue, corrigée et augmentée. Paris, Bobé et Hingray.

DAVID, Jules (1853) : Notice sur les ouvrages de M. Jules David (helléniste) concernant la langue grecque. Paris, Imprimerie de L. Marinet.

DAVID, Jules (Inédit) : Dictionnaire français et grec ancien.

Dictionnaire de biographie française(1965). Paris, Letouzay et Ané.

Grand dictionnaire universel du XIX ${ }^{e}$ siècle(1990). Larousse, Pierre Nîmes C. Lacour (réimpression de l'édition 1866/1876).

FENELON(1717) : Les aventures de Télémaque, fils d'Ulysse. Paris, J. Estienne.

HUGO, Victor (1828) : Les Orientales, in Oeuvres poétiqes complètes, réunies et présentées par Francis Bouvet. Paris, Jean-Jacques Pauvert.

LANCASTER, Joseph (1805): Improvements in Education, as it respects the industrious classes of the Community, containing among other important particulars, An Account of the Institution for the Education of One Thousand Poor Children, Borrough Road, Southwark; and of the New System of Education on which it is conducted. Third Edition with additions. Londres, Barton and Harvey.

LEGRAND, Emile (1878) : Grammaire grecque moderne, suivie du Panorama de la Grèce d'Alexandre Soutsos. Paris, Maisonneuve.

LEGRAND, Emile (1906) : Bibliographie hellénique ou Description raisonnée des ouvrages publiés par des Grecs aux $X^{e}$ et XVI ${ }^{e}$ siècle. T. IV, Paris.

Logios Ermis(Le Mercure Savant) : (1821), Vienne.

LÜDEMANN, Wilhem von (1826) : Lehrbuch der neugriechischen Sprache. Leipzig.

Megali elliniki engyklopaideia(1957-1964), Athènes, FOINIX, $2^{\mathrm{e}}$ édition. 
MITCHELL, John (1824) : A Grammatical parallel of the Ancient and Modern Greek languages [trad, anglaise du Parallèle des langues grecques ancienne et moderne de J. David]. Londres, printed for the Author.

PALMADE, Guy (1979) : Les méthodes en Pédagogie. Paris, PUF, Que Sais-je?

PROVATA, Despina (2001) : « Un témoignage tardif des Lumières en Grèce : Le dictionnaire inédit de Jules David », in Comparaison, n. 12, 82-87.

SCHINAS, Michel (1829) : Grammaire élémentaire du Grec moderne divisée en deux parties, la première contenant la théorie complète de la prononciation et de la prosodie, avec les règles, [...], Paris, Hachette.

SCHMIDT, J. A. Erdmann (1808) : Neugriechische Sprachlehre. Leipzig. Joachim's Literarischen Magazin.

SIMOPOULOS, Kyriakos (1975) : Xenoi taxidiotes stin Ellada, 1810-1821, t. $3^{2}$. Athènes [par l'auteur].

SOUTSOS, Panayotis (1828) : Odes d'un Jeune Grec. Paris, Emler frères.

TRIANDA FYLLIDIS, Manolis (1938) : Neoelliniki Grammatiki. Istoriki Eisagogi. Athènes, Dimitrakou.

TROYAT, Henri (1990) : La femme de David. Paris, Flammarion.

VOLNEY, C.-F. (1821) : Considérations sur la guerre des Turks in CEuvres complètes, t.3. Paris, Bossange.

WINNOCK, George (1825) : The modem Greek Grammar of Julius David formerly one of the Professors of the Greek College of Scio. Translated from the original French by [...] Londres, Published for the Translator, by J. Souter.

\section{NOTES}

1. Il existe très peu d'informations sur Jules David, en particulier sur son séjour à Chio, une grande partie des archives de l'île ayant été détruite pendant les massacres de 1822 et les tremblements de terre de 1881 . Le petit nombre de documents inédits le concernant que j'ai réussi à obtenir seront annexés à l'essai sur la vie et l'œuvre de l'helléniste que je prépare.

2. Chio est une des sept villes qui se disputent l'honneur d'être la patrie d'Homère.

3. Voir sa lettre à Coray du 23 février 1817 (Coray $1984: 17$ - 18).

4. Selon d'autres (revue Armonia 1900: 173, K. Simopoulos 1975 : 535), Jules s'est marié à Smyrne.

5. La recommandation de Coray à ses compatriotes d'augmenter le salaire du Français Mendouze, le «maître d'école » qui remplaça David au Gymnase et de lui trouver une épouse, afin qu'il ne nous quitte «lui aussi » (Coray 1984: 107), me fait penser que Jules n'était pas satisfait de ses appointements et qu'à Chio il était encore célibataire.

6. Selon sa mère "à Smyrne, il y traitait des affaires auxquelles je ne comprenais rien et apprenait le grec. Tout cela ne le nourrissait guère. Il fallait que nous l'aidions, lui aussi, à garnir sa marmite. » (Cité dans Troyat $1990: 180$ ).

7. Aux archives de la Sorbonne cependant son nom ne figure pas dans la liste des enseignants de la Faculté des lettres.

8. Par contre, son fils, le baron Jérôme David (1823 - 1882), renia vite les idéaux de son grandpère et de son père et devint un des politiciens les plus en vue et les plus réactionnaires du Second Empire.

9. Tous les Français cultivés, en particulier ceux qui comparaient la Grèce classique avec celle de la fin du XVIIIe siècle, ne partageaient pas les sentiments des philhellènes. Volney, par exemple, jugeait le peuple grec abâtardi et ajoutait que « pour ressusciter les Grecs anciens, il faudra 
rendre des mœurs aux Grecs modernes, devenus la race la plus vile et la plus corrompue de l'univers [...]» (Volney 1821: 431).

10. Sur le Dictionnaire de Jules David voir l'article de Provata (2001).

11. Manolis Triandafyllidis (1883-1959), éminent linguiste grec ; Michel Schinas (1792-1870), Grec de Constantinople, polyglotte, érudit, politicien, auteur d'une Grammaire élémentaire du Grec moderne [...] ci l'usage des Français (1829). Dans la préface, il reconnaît l'utilité du manuel de David pour l'enseignement « du langage familier ou vulgaire » et trouve « en général bien de suivre ce guide » mais le juge moins satisfaisant pour l'enseignement de « la prose des meilleurs auteurs » et des «journaux grecs. » Il lui reproche également de référer l'apprenant « à chaque instant à la grammaire du grec ancien»; Emile Legrand (1841-1903) helléniste français, auteur de la monumentale Bibliographie hellénique (1906) et d'une Grammaire grecque moderne [...] (1878), où il rend hommage aux « excellents travaux de M. Jules David» (X).

12. Selon Troyat (1990 : 147), Jules a étudié à l'université de Göttingen. Lui-même, dans sa demande [document inédit] d'une " place de Membre du Conseil de l'Instruction publique», datée du 18 août 1831, il affirme aussi avoir étudié à l'université de Göttingen et y avoir « suivi les cours du célèbre Heyne. » Toutefois, le professeur Düwell dans une communication écrite, pour laquelle je le remercie, m'informe que le nom de Jules David ne figure ni dans les registres des étudiants de l'Université ni dans la liste des élèves du professeur Heyne.

13. David ne partage pas l'avis des érudits européens sur la prononciation du grec ancien. Étant donné que personne aujourd'hui ne sait avec certitude comment cette langue était parlée par les Grecs éduqués à l'époque classique, il préfère aux spéculations des savants étrangers la prononciation utilisée par les Grecs modernes instruits, car elle jouit d'une longue tradition domestique et son ancienneté est attestée par nombre de documents de l'Antiquité (1820:_).

14. David cite comme exemple le Neugriechische Sprachlehre d'Erdmann Schmidt, publié à Leipzig en 1808. Il affirme en avoir pris connaissance après avoir rédigé sa Méthode, ce qu'il ne regrette pas cet ouvrage n'étant qu'un « mélange confus des principes et des formes de l'hellénique et du grec moderne, un chaos indigeste [...] » qui rend l'étude de cette langue déjà « assez difficile par elle-même »encore plus difficile (1821b : iv - v).

15. Devenu collège de type européen, il était réputé dans tous les Balkans et fréquenté par des élèves grecs et étrangers, surtout depuis que Neofytos Vamvas (1776-1855), protégé et assistant de Coray à Paris (1808-1815, grand érudit et un des« didaskaloi tou genous » [précepteurs de la nation], en fut nommé directeur.

16. II s'agit de l'enseignement mutuel introduit en Angleterre en 1798 par Bell et Lancaster. « Les écoliers les plus âgés et les plus capables deviennent des moniteurs et font office de maîtres vis-àvis de leurs camarades plus-jeunes ou moins avancés (monitorial system) [...] (Palmade, 1979 : 87). 17. Joseph Lancaster (1805: 23) : «THE Boys' School was instituted as a Free School by Joseph Lancaster, in 1801 ; and is actually extended to Seven Hundred Boys, who are instructed upon a Plan entirely new ; by means of which, ONE MASTER alone can educate One Thousand Boys, in Reading, Writing, and Arithmetic, as effectually, and with as little Trouble, as Twenty or Thirty have ever been instructed by the usual modes of Tuition. "

18. II est difficile de dire si les craintes de Vamvas étaient réelles ou si elles n'étaient qu'un prétexte pour imposer dans son école ses propres principes pédagogiques qui n'étaient pas exactement ceux de Coray.

19. Dictionnaire de Biographie Française (1965), Megali Elliniki Engyklopaideia (1957). 


\section{RÉSUMÉS}

Dans mon exposé, j'esquisse la vie de l'helléniste Jules David (1783-1854) et j'examine comment il a enseigné le français en Grèce, encore occupée par les Turcs. Je décris également ses ouvrages sur les rapports du grec ancien avec le grec moderne et j'évalue leur importance pour la philologie et la didactique des langues.

In my presentation I outline the life of the hellenist Jules David (1783-1854) and examine the way he taught French in Greece, still under Turkish rule. I also describe his works on the relationship between ancient and modern Greek and I evaluate their importance for philology and language teaching.

\section{INDEX}

Keywords : Jules David, biography, Hellenism Greece, XIXth century

Mots-clés : Jules David, biographie, hellénisme, Grèce, XIXe siècle

\section{AUTEUR}

\section{JEAN ANTOINE CARAVOLAS}

Montréal 